\title{
Nematic Ordering Pattern Formation in the Process of Self-Organization of Microtubules in a Gravitational Field
}

\author{
Hu Jian • Qiu Xijun • Li Ruxin
}

Received: 9 June 2006 / Accepted: 22 December 2006 /

Published online: 1 February 2007

(C) Springer Science + Business Media B.V. 2007

\begin{abstract}
Papaseit et al. (Proc. Natl. Acad. Sci. U.S.A. 97, 8364, 2000) showed the decisive role of gravity in the formation of patterns by assemblies of microtubules in vitro. By virtue of a functional scaling, the free energy for MT systems in a gravitational field was constructed. The influence of the gravitational field on MT's self-organization process, that can lead to the isotropic to nematic phase transition, is the focus of this paper. A coupling of a concentration gradient with orientational order characteristic of nematic ordering pattern formation is the new feature emerging in the presence of gravity. The concentration range corresponding to a phase coexistence region increases with increasing $g$ or MT concentration. Gravity facilitates the isotropic to nematic phase transition leading to a significantly broader transition region. The phase transition represents the interplay between the growth in the isotropic phase and the precipitation into the nematic phase. We also present and discuss the numerical results obtained for local MT concentration change with the height of the vessel, order parameter and phase transition properties.
\end{abstract}

Key words microtubules $\cdot$ gravitational field $\cdot$ concentration gradient $\cdot$ nematic ordering

\section{Introduction}

Microtubules (MTs) are long rigid hollow cylindrical shells of about $25 \mathrm{~nm}$ diameter, formed by a regular helical lattice of $\alpha-\beta$ tubulin dimers, noncovalently joined by protein-protein bonds, which can be viewed as constructed of 13 parallel protofilaments joined laterally. Their length can vary from tens of nanometers to hundreds of microns [2]. MTs are important components and functional units in cytoskeletons, as well as cellular organization and

H. Jian $(\triangle) \cdot$ Q. Xijun

Department of Physics, Shanghai University, Shanghai 200444, China

e-mail: shhujian2005@yahoo.com.cn

L. Ruxin

Shanghai Institute of Optics and Fine Mechanics, Chinese Academy of Science, Shanghai 201800, China 
information processing [3-5]. MTs play a crucial role in several vital processes such as intracellular transport, cell division, motility and determination of cell shape [6].

Before we discuss the influence of the gravitational field on the process of selforganization of MTs, it is worthwhile to get a rough estimate of the effect of gravity on the MT system. For a MT, its linear density is $1.6 \times 10^{5}(\mathrm{~g} / \mathrm{mol}) / \mathrm{nm}$, the molecular mass of a tubulin dimer, subunit of a MT, is $m_{0} \sim 10^{5} \mathrm{~g} / \mathrm{mol}$. The influence of the gravitational field is significant if the gravitational potential energy of the system is on the order of, or larger than, the thermal energy, $k T$, that is $(m g h / k T) N=\beta N \geq 1$, where $m=m_{0}-\rho v$ is the mass of a tubulin corrected for buoyancy $\left(\rho \sim 1 \mathrm{~g} / \mathrm{cm}^{3}\right.$ and $v \sim 100 \mathrm{~nm}^{3}$ are the density of water and the volume of the tubulin dimer, respectively), $k$ is the Boltzmann constant and $N$ denotes the number of tubulin dimers in a MT. For vessel height $h=1 \mathrm{~cm}$, the gravitational acceleration $\mathrm{g} \sim 10 \mathrm{~m} / \mathrm{s}^{2}$ and room temperature $(T \sim 300 \mathrm{~K})$ conditions, if we take the MT length to be $L=1 \mu \mathrm{m}$, then one can get $\beta N \sim 1$. Therefore, gravity can influence the equilibrium properties of the MT system even for the earth's gravitational field.

Gravity is known to crucially affect biological systems [7-9]. In fact, it can cause the isotropic symmetry breaking that is necessary for pattern formation during animal development and cell morphogenesis. Symmetry breaking is an essential feature of embryo development. An example is the early stages of frog embryo development $[10,11]$, in which, starting from a spheroidal shape, differentiation between the top and bottom and left and right of the embryo occurs. During these events, there is a striking interplay between morphogenetic development and the effects of gravity [12, 13]. MT arrays play a critical role in these processes [14, 15], and they have been reported as forming "in vitro" dissipative structures, which might play an important role in morphogenesis. In addition, the gravity effect might provide the underlying explanation for some aspects of biological gravitropism, such as occurs in plants [16-18]. Recent space-flight experiments performed by Tabony's team [8] provided further evidence that a microgravity environment strongly affects the self-organization of MT assemblies.

It is known that in a system of homogenously long rodlike molecule solution, the excluded volume effect can force the isotropic rodlike molecules to line up parallel to each other and a long-range orientational order characteristic of nematic ordering [19] takes place when their concentration exceeds a critical number, namely $C_{\text {cri }} \sim L^{-2} d^{-1}$ [20], where $L$ and $d$ are the length and diameter for long rods. There are several approaches related to the calculation of the isotropic-nematic phase transition for these systems. However, most of these approaches did not take gravitational effects into consideration. In fact, this transition always occurs in a gravitational field [21]. The gravitational field can serve as a driving force and make the rods concentrated near the bottom of the vessel and sometimes induce nematie ordering. In contrast to common rodlike objects, biological self-assembled objects such as MTs have considerable molecular concentration and mass, sufficient to induce a significant concentration gradient in a gravitational field. This concentration inhomogeneity within the volume can provoke the isotropic-nematic phase transition.

In [21], the authors, using the Onsager approximation, theoretically investigated the isotropic to nematic transition properties of sufficiently dilute solutions of long rods in a gravitational field. In this paper, we treat the MT as a long rigid rodlike molecules, and by virtue of a functional scaling method [22] we constructed the free energy for a MT solution system with a high concentration in the gravitational field and theoretically studied the influence of the gravitational field on the nematic ordering in this system. The paper is organized as follows. In Section 2, we describe the theoretical model by constructing the free energy for the MT system subject to a gravitational field and gives both the phase's equilibrium concentration distribution function and the coexistence relation at the phase 
boundary. In Section 3, we discuss the numerical results obtained for phase diagrams, MT concentration distribution diagrams and MT orientational order and we end with some concluding remarks in Section 4.

\section{Theoretical Model}

We consider the solution of MT living polymers in a vessel subject to a gravitational field. Suppose that the gravitational field acts in the $z$ direction and put the origin of coordinates at the bottom of the reservoir $(z=0)$. Further, let us divide the volume of the vessel $V$ into the very large number of identical layers aligned perpendicularly to the gravitational field. With such division, the local MT number concentration $C(\xi)$ has the same value within each layer. We define $d N_{z}(\Omega)=N_{z} f_{z}(\Omega) d \Omega$ as the number of MTs in the layer $z$ with axis directions within the small spatial angle $d \Omega, N_{Z}$ is the total number of MTs in the layer $z$, and $\xi=z / h$ represents the dimensionless height. Let $f_{z}(\Omega)$ be the orientational distribution function of one MT in the layer $z$, and it is normalized as $\int f_{z}(\Omega) d \Omega=1$.

In contrast to a sufficiently dilute rods solution, the excluded volume effects are very important for a MT solution system with a high concentration due to the concentration and significant size of MT. So we introduce the following functional scaling transformation [22] with the molecular shape anisotropy for any packing function $J(\eta)$ via the excluded volume of two rigid cylindrical MTs

$$
J(\eta) \rightarrow J(\eta) \frac{1}{8}<\bar{v}_{\text {excl }}\left(\Omega_{1}, \Omega_{2}\right)>
$$

where $\bar{v}_{\text {excl }}\left(\Omega_{1}, \Omega_{2}\right)=v_{\text {excl }}\left(\Omega_{1}, \Omega_{2}\right) / v_{0}, v_{0}$ is the volume of a hard sphere, $\eta=C(\xi) v_{0}$ is the packing fraction, and $\Omega_{1}$ and $\Omega_{2}$ specify the orientations of two axiosymmetric MTs. Here $<>$ denotes the orientational average. The choice of the factor $1 / 8$ is due to $\bar{v}_{\text {excl }}=8 v_{0}$ for a hard sphere.

For two MT cylinders with orientations, $\Omega_{1}$ and $\Omega_{2}$, respectively,

$$
\left\langle\overline{\mathrm{v}}_{\mathrm{excl}}\left(\Omega_{1}, \Omega_{2}\right)\right\rangle=8+3 \pi \delta \rho(\xi)
$$

where $\delta=(L / d)^{2}[\pi(1+3 L / 2 d)]^{-1}$. Here $\rho(\xi)$ describes the angle-dependent excluded volume effects given by

$$
\rho(\xi)=\frac{4}{\pi} \iint\left|\sin \gamma\left(\Omega_{1}, \Omega_{2}\right)\right| f\left(\Omega_{1}, \xi\right) f\left(\Omega_{2}, \xi\right) d \Omega_{1} d \Omega_{2}
$$

where $L$ and $d$ denote the length and diameter of a MT, and $\gamma$ is the angle between $\Omega_{1}$ and $\Omega_{2}$.

Consequently, the local free energy of the layer $\xi$ for a system of rigid spherocylindrical MTs may take the form

$$
\frac{\varpi(\xi)}{k T}=\ln C(\xi)+\sigma(\xi)+\frac{\eta(4-3 \eta)}{(1-\eta)^{2}}\left(1+\frac{3 \pi \delta}{8} \rho(\xi)\right)+\int \beta \xi f(\Omega, \xi) d \Omega .
$$

The first term describes the entropy of a translational motion; the second term is the orientational entropy of the layer $\xi$

$$
\sigma(\xi)=\int f(\Omega, \xi) \ln (4 \pi f(\Omega, \xi)) d \Omega
$$

the third term represents the steric interaction between MTs in the second virial approximation, and the last term is the average potential energy of a MT in the gravitational field, where $\beta=m g h / k T$. 
The free energy of the whole MT system is a sum of free energies of all layers. Let the number of layers become very large, and then the sum can be replaced by the following integral

$$
\begin{aligned}
\frac{F}{V k T} & =\int_{0}^{1} d \xi C(\xi) \frac{\varpi(\xi)}{k T} \\
& =\int_{0}^{1} d \xi C(\xi)\left[\ln C(\xi)+\sigma(\xi)+\frac{\eta(4-3 \eta)}{(1-\eta)^{2}}\left(1+\frac{3 \pi \delta}{8} \rho(\xi)\right)+\beta \xi\right]
\end{aligned}
$$

Once the MT concentration exceeds the critical value $\left(C(\xi)>C_{\text {cri }}\right)$, the possibility that nematic phase will appear at the bottom of the vessel and isotropic phase on top in a gravitational field should be considered. We denote the height of the phase boundary position in the vessel as $\xi=x$, so the volumes occupied by the nematic and isotropic phases are $V_{n}=x V$ and $V_{i}=(1-x) V$, respectively. In this case, the free energy of the whole system becomes

$$
\frac{F}{V k T}=\int_{0}^{x} d \xi C_{n}(\xi) \frac{\varpi_{n}(\xi)}{k T}+\int_{x}^{1} d \xi C_{i}(\xi) \frac{\varpi_{i}(\xi)}{k T}
$$

where

$$
\frac{\varpi_{n}(\xi)}{k T}=\ln C_{n}(\xi)+\sigma_{n}(\xi)+\frac{\eta_{n}\left(4-3 \eta_{n}\right)}{\left(1-\eta_{n}\right)^{2}}\left(1+\frac{3 \pi \delta}{8} \rho_{n}(\xi)\right)+\beta \xi
$$

is the local free energy of the nematic phase, and

$$
\frac{\varpi_{i}(\xi)}{k T}=\ln C_{i}(\xi)+\sigma_{i}(\xi)+\frac{\eta_{i}\left(4-3 \eta_{i}\right)}{\left(1-\eta_{i}\right)^{2}}\left(1+\frac{3 \pi \delta}{8} \rho_{i}(\xi)\right)+\beta \xi
$$

is the local free energy of the isotropic phase. Here the indices $i$ and $n$ designate the isotropic phase and the nematic phase respectively.

The equilibrium distribution function $f(\Omega, \xi)$ can be determined at a given packing fraction $\eta$ by minimizing the functional (7) with respect to this function. However, this will lead to a rather complicated nonlinear integral equation. In the case where the volume contains two phases separated by a phase boundary $f(\Omega, \xi)$ is discontinuous at the boundary and the function $f_{n}(\Omega, \xi)$ in the anisotropic phase is quite different from that in the isotropic one.

In the isotropic phase $\sigma_{i}=0, \rho_{i}=1$ and the orientational distribution function becomes $f_{i}(\xi)=\frac{1}{4 \pi}$, so (9) can be simplified as

$$
\frac{\varpi_{i}(\xi)}{k T}=\ln C_{i}(\xi)+\frac{\eta_{i}\left(4-3 \eta_{i}\right)}{\left(1-\eta_{i}\right)^{2}}\left(1+\frac{3 \pi \delta}{8}\right)+\beta \xi .
$$

For the sake of simplicity, the orientational distribution function $f_{n}(\Omega, \xi)$ in the nematic phase can be obtained by applying an approximate variational method with a trial function depending on the variational parameter $\alpha(\xi)$ whose form is

$$
f_{n}(\Omega, \alpha(\xi))=\frac{\alpha(\xi)}{4 \pi} \begin{cases}e^{-\frac{\alpha(\xi) \theta^{2}}{2}}, & 0<\theta<\pi / 2 \\ e^{-\frac{\alpha(\xi)(\pi-\theta)^{2}}{2}}, & \pi / 2<\theta<\pi .\end{cases}
$$


Substituting $f_{n}(\Omega, \alpha(\xi))$ into (8) and minimizing the equation with respect to $\alpha(\xi)$, one can obtain:

$$
\frac{d \sigma_{n}(\alpha)}{d \alpha}+\frac{\eta_{n}\left(4-3 \eta_{n}\right)}{\left(1-\eta_{n}\right)^{2}} \frac{3 \pi \delta}{8} \frac{d \rho_{n}(\alpha)}{d \alpha}=0
$$

So the orientational entropy can be evaluated:

$$
\sigma_{n}(\alpha(\xi))=\int f_{n}(\Omega, \alpha(\xi)) \ln \left\{\left(4 \pi f_{n}(\Omega, \alpha(\xi))\right) d \Omega \approx \ln \alpha(\xi)-1\right.
$$

and the dimensionless second virial coefficient in the nematic phase is found to be

$$
\rho_{n}(\xi)=\frac{4}{\pi} \iint\left|\sin \gamma\left(\Omega_{1}, \Omega_{2}\right)\right| f_{n}\left(\Omega_{1}, \alpha(\xi)\right) f_{n}\left(\Omega_{2}, \alpha(\xi)\right) d \Omega_{1} d \Omega_{2} \approx \frac{4}{\sqrt{\pi \alpha(\xi)}} .
$$

Substituting (13) and (14) back into (12), one can obtain

$$
\sqrt{\alpha(\xi)}=\frac{\eta_{n}\left(4-3 \eta_{n}\right)}{\left(1-\eta_{n}\right)^{2}} \frac{3 \delta \sqrt{\pi}}{4}
$$

With this, and based on (8), the local free energy of the nematic phase is given as

$$
\frac{\varpi_{n}(\xi)}{k T}=\ln C_{n}(\xi)+2 \ln \frac{2 C_{n}(\xi)}{\sqrt{\pi}}+\frac{\eta_{n}\left(4-3 \eta_{n}\right)}{\left(1-\eta_{n}\right)^{2}}+1+\beta \xi
$$

The chemical potentials in the phases are

$$
\mu_{n, i}(\xi)=\varpi_{n, i}(\xi)+\frac{p_{n, i}(\xi)}{C_{n, i}(\xi)}
$$

and the osmotic pressures $p_{n, i}(\xi)$ in the layer $\xi$ are

$$
p_{n, i}(\xi)=-\frac{\partial \varpi_{n, i}(\xi)}{\partial \frac{1}{C_{n, i}(\xi)}}=\left[C_{n, i}(\xi)\right]^{2} \frac{\partial \varpi_{n, i}(\xi)}{\partial C_{n, i}(\xi)} .
$$

Consequently, the osmotic pressures in the isotropic and nematic phases are given as follows:

$$
\begin{aligned}
& p_{i}(\xi)=\left\{C_{i}(\xi)+2 v_{0}\left[C_{i}(\xi)\right]^{2}\left(1+\frac{3 \pi \delta}{8}\right) \frac{\left(v_{0} C_{i}(\xi)-2\right)}{\left(v_{0} C_{i}(\xi)-1\right)^{3}}\right\} k T, \\
& p_{n}(\xi)=\left\{C_{n}(\xi)+\sqrt{\pi} C_{n}(\xi)+2 v_{0}\left[C_{n}(\xi)\right]^{2} \frac{\left(v_{0} C_{n}(\xi)-2\right)}{\left(v_{0} C_{n}(\xi)-1\right)^{3}}\right\} k T .
\end{aligned}
$$


Correspondingly, from (10), (16), (17) and (18) we can obtain the following chemical potentials:

$$
\begin{aligned}
\frac{\mu_{i}(\xi)}{k T}= & \ln C_{i}(\xi)+\frac{\eta_{i}\left(4-3 \eta_{i}\right)}{\left(1-\eta_{i}\right)^{2}}\left(1+\frac{3 \pi \delta}{8}\right)+\beta \xi+1 \\
& +2 v_{0} C_{i}(\xi)\left(1+\frac{3 \pi \delta}{8}\right) \frac{\left(v_{0} C_{i}(\xi)-2\right)}{\left(v_{0} C_{i}(\xi)-1\right)^{3}}
\end{aligned}
$$

and

$$
\begin{aligned}
\frac{\mu_{n}(\xi)}{k T}= & \ln C_{n}(\xi)+2 \ln \frac{2 C_{n}(\xi)}{\sqrt{\pi}}+\frac{\eta_{n}\left(4-3 \eta_{n}\right)}{\left(1-\eta_{n}\right)^{2}}+2+\beta \xi+\sqrt{\pi} \\
& +2 v_{0} C_{n}(\xi) \frac{\left(v_{0} C_{n}(\xi)-2\right)}{\left(v_{0} C_{n}(\xi)-1\right)^{3}} .
\end{aligned}
$$

It is known that in stable equilibrium the chemical potential is the same in both phases and does not depend on height. So the equilibrium local MT concentrations satisfy the following condition:

$$
\frac{d p_{n, i}(\xi)}{d \xi}=-m g h C_{n, i}(\xi)
$$

Substituting (19) and (20) into (23) respectively, one can obtain the equilibrium local concentrations in both phases as follows:

$$
C_{n}(\xi) \approx \frac{C_{n}}{I_{1}(x)} e^{-\frac{(\beta \xi+3)}{1+\sqrt{\pi}}},
$$

and

$$
C_{i}(\xi) \approx \frac{C_{i}}{I_{2}(x)} e^{-\left(\beta \xi+3+\frac{9 \pi \delta}{8}\right)} .
$$

where $C_{n}$ and $C_{i}$ are dimensionless average MT concentrations in the nematic and isotropic phases, respectively. $I_{1}(x)=\int_{0}^{x} e^{-\frac{(\beta \xi+3)}{1+\sqrt{\pi}}} d \xi$ and $I_{2}(x)=\int_{x}^{1} e^{-\left(\beta \xi+3+\frac{9 \pi \delta}{8}\right)} d \xi$ are the normalization factor.

The equilibrium corresponds to the equality of osmotic pressures and chemical potentials at the boundary $\xi=x$, that is

$$
\left\{\begin{array}{l}
p_{n}(x)=p_{i}(x) \\
\mu_{n}(x)=\mu_{i}(x)
\end{array}\right.
$$

Using the expressions in (19), (21) and (25) for the isotropic phase and (20), (22) and (24) for the nematic phase and letting $C_{n}(x)=\frac{C_{n}}{I_{1}(x)} e^{-\frac{(\beta x+3)}{1+\sqrt{\pi}}}$ and $C_{i}(x)=\frac{C_{i}}{I_{2}(x)} e^{-\left(\beta x+3+\frac{9 \pi \delta}{8}\right)}$, the coexistence relations (26) at the boundary can be written as:

$$
\left\{\begin{array}{c}
(1+\sqrt{\pi}) C_{n}(x)+2 v_{0}\left[C_{n}(x)\right]^{2} \frac{\left(v_{0} C_{n}(x)-2\right)}{\left(v_{0} C_{n}(x)-1\right)^{3}}=C_{i}(x)+2 v_{0}\left[C_{i}(x)\right]^{2}\left(1+\frac{3 \pi \delta}{8}\right) \frac{\left(v_{0} C_{i}(x)-2\right)}{\left(v_{0} C_{i}(x)-1\right)^{3}} \\
\ln C_{n}(x)+2 \ln \frac{2}{\sqrt{\pi}} C_{n}(x)+1+\sqrt{\pi}+\frac{v_{0} C_{n}(x)\left(4-3 v_{0} C_{n}(x)\right)}{\left(1-v_{0} C_{n}(x)\right)^{2}}+2 v_{0} C_{n}(x) \frac{\left(v_{0} C_{n}(x)-2\right)}{\left(v_{0} C_{n}(x)-1\right)^{3}} \\
=\ln C_{i}(x)+\frac{v_{0} C_{i}(x)\left(4-3 v_{0} C_{i}(x)\right)}{\left(1-v_{0} C_{i}(x)\right)^{2}}\left(1+\frac{3 \pi \delta}{8}\right)+2 v_{0} C_{i}(x)\left(1+\frac{3 \pi \delta}{8}\right) \frac{\left(v_{0} C_{i}(x)-2\right)}{\left(v_{0} C_{i}(x)-1\right)^{3}}
\end{array}\right.
$$


Fig. 1 Phase diagram of MT living polymers in a gravitational field. The dimensionless total average MT concentration is plotted against the dimensionless parameter associated with the gravitational field $\beta=m g h / k T$, for $L=5 \mu \mathrm{m}$. Here $I$ denotes the isotropic phase and $N$ is the nematic phase, $I+N$ corresponds to the phase coexistence (separation) region

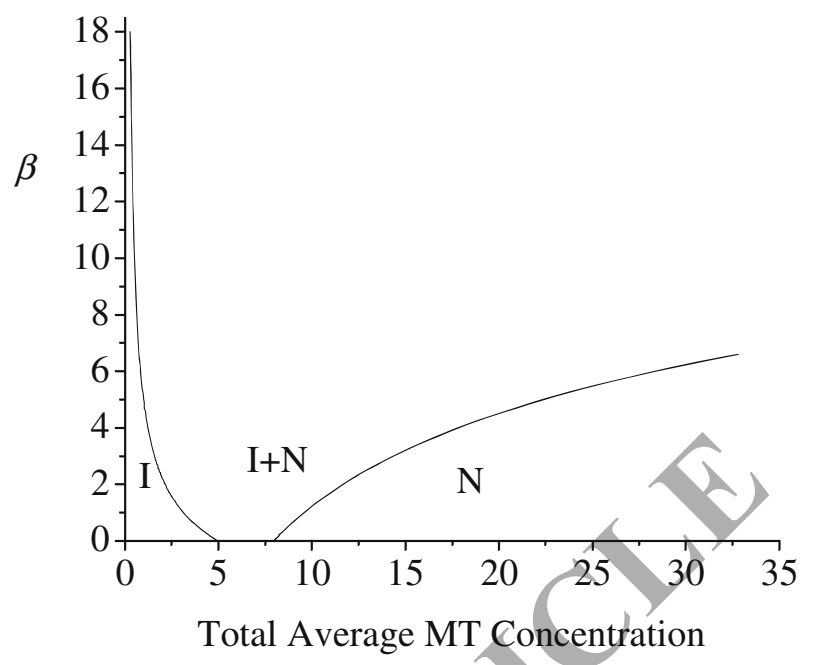

\section{Numerical Results and Discussion}

In (27), we put $x=0$ for the onset of the isotropic phase and $x=1$ for the onset of the nematic phase, and numerically solve (27) along with the conservation of mass condition, $C=C_{n}+C_{i}$. This gives the values of average dimensionless MT concentrations in the isotropic phase $C_{i}$ and nematic phase $C_{n}$ coexisting at equilibrium. The boundaries of the coexistence region can also be obtained. Figure 1 gives a typical phase diagram in the variables $C$ (the dimensionless total average MT concentration) and the dimensionless parameter $\beta$. This diagram reveals that gravity facilitates the isotropic to nematic phase transition and significantly broadens the transition region. The phase transition represents the interplay between the growth in the isotropic phase and the precipitation into the nematic phase. In addition, when increasing $\beta$ or MT concentration, the phase coexistence region becomes broader.

Fig. 2 The dimensionless local MT concentration $C(\xi)$ is plotted as a function of the dimensionless height $\xi$ at a fixed value of $\beta$. The solid lines refer to $\beta=6.4$; the dashed lines refer to $\beta=2.4$, the dimensionless total MT concentration is $C=7.8$, and $L=5 \mu \mathrm{m}$

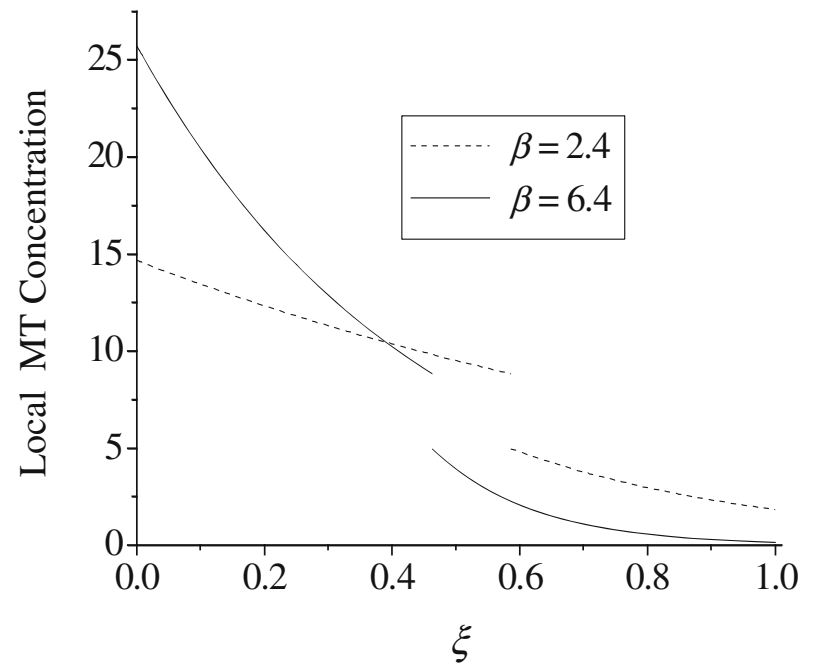


Figure 2 is a plot of the local MT concentration as a function of the height $\xi$ at a fixed value of $\beta$. This plot shows that gravity coupled with the interactions between MTs forms the gradient distribution of MT concentration with height $\xi$. With the increase of $\beta$, the local MT concentration at the bottom of the vessel gradually increases while on top it gradually decreases. Both of the phase concentrations in the boundary layer coincide with that in the absence of the gravitational field. This is the case because all the MTs within a given layer are subject to the same gravitational potentials, and the MT concentrations are discontinuous at the phase boundary.

The phase boundary position $x$ vs $\beta$ for different values of total MT concentration is shown in Fig. 3. It demonstrates that when the total MT concentration is low (close to the isotropic phase boundary), the volume of the nematic phase is low. The increasing gravity induces the increase of the nematic phase until, exhausting the isotropic phase at $\beta \sim 2$, the increase of the nematic phase stops. After this, the volume of nematic phase gradually decreases under gravity, indicating the sinking of the MT aggregates in the nematic phase, and the MT concentration at the bottom of the vessel becomes larger. If the total MT concentration is high (close to the nematic phase boundary), the MT aggregates in the nematic phase can only shrink from the top of the vessel with increasing gravity, and the phase boundary position slightly decreasing.

In the nematic phase, the orientational order changes with height $\xi$ can be described by an order parameter $S(\xi)$ given by $S(\xi)=\int P_{2}(\cos \alpha(\Omega, \xi)) f(\Omega, \xi) d \Omega$, where $P_{2}(\cos \alpha(\Omega, \xi))$ is the second Legendre polynomial, while in the isotropic phase the order parameter $S(\xi)$ falls to zero.

For MTs in an initially isotropic distribution, in an orientation which maximizes the orientational entropy, gravity, coupled with geometric exclusion effects, leads to a longrange orientational order characteristic of a polar nematic liquid crystal (LQ) phase appeared at the bottom of the vessel. The idea of a LQ representation of microtubules was first postulated by authors of [23]. Obviously, LQ properties of MTs will affect their alignment along the surface of the cell. For long MT cylinders, condensing many MT ends at the surface carries a high entropy cost and is therefore unlikely to occur. This is consistent with the experimental fact mentioned in [24] that MTs are obliquely aligned parallel to the cell wall corresponding to the largest area $(40 \times 10) \mathrm{mm}^{2}$ and pointing toward

Fig. 3 The position of the phase boundary $x$ plotted as a function of $\beta$ for three different values of the dimensionless total MT concentration: $C=7.8$ (solid line), $C=7$ (dashed line) and $C=6$ (dotted line); $L=5 \mu \mathrm{m}$

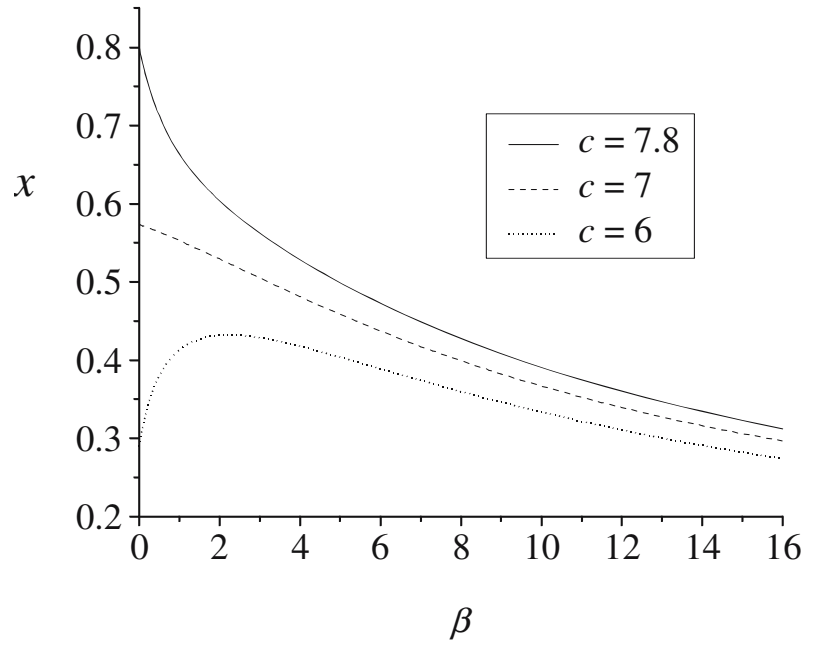


the smallest surface area. This significantly reduces the configurational entropy of one MT in contact by $\Delta S=k_{B} \ln \left(\frac{L}{d}\right) \approx 5.3 k_{B}$ [24]. These gravity-dependent MT arrays play an important role in cell morphogenesis and, as mentioned above, possibly provides an additional explanation for biological gravitropism, such as occurs in plants.

\section{Conclusions}

Although the gravitational field is often neglected in the description of physicochemical processes, its influence on biological heavy objects such as MTs is noticeable and important because this influence on biological living polymers is much stronger than that for nonaggregating particles [25]. Moreover, this always occurs even under normal earth gravity conditions.

In the present paper, we theoretically studied the isotropic to nematic phase transition of MT system in a gravitational field. This transition shows that MT's self-organization process is very sensitive to the gravitational field. Gravity, which can induce concentration inhomogenity, does not only facilitate the formation of the nematic phase at the bottom of the vessel, but it also seriously broadens the phase coexistence region. In the low concentration limit, it is easy to obtain an expression for the free energy of the rigid rods system given by authors in [21]. Therefore, for a sufficiently dilute solution of rigid rods, our numerical results agree with those of [21].

In the experiments of Tabony et al. [8], striped patterns of MTs in the cell with dimensions $40 \times 10 \times 1 \mathrm{~mm}^{3}$ appear under gravity [9] (on earth and in flight under $1 \mathrm{~g}$ centrifugation), with two adjacent stripes made up of highly oriented MT bundles at angles of $45^{\circ}$ and $135^{\circ}$, respectively, to the horizontal. Our theoretical results are consistent with this experimental observation that the earth gravity can crucially affect the self-organization process of MT system. While under microgravity conditions $\left(2 \times 10^{-4} \mathrm{~g}\right)$ [1], MTs show no specific pattern formation; this is in accordance with our results that the influence of the gravitational field is significant if the gravitational potential energy of the system is on the order of, or larger than, the thermal energy $k T$, that is $(m g h / k T) N=\beta N \geq 1$. So under microgravity conditions $\beta N \sim 10^{-4} \ll 1$, the effects of microgravity are negligible. In the experiments of Tabony et al., the average length of MTs is approximately $L=5 \mu \mathrm{m}$ and the tubulin concentration is $10 \mathrm{mg} / \mathrm{ml}$, roughly $70 \%$ of which is assembled into MTs. Thus, the MT concentration is very close to the nematic phase boundary which we have obtained in this paper, and the $C_{\mathrm{TE}}^{\mathrm{MT}}=3 \times 10^{18} \mathrm{~m}^{-3}>C_{\mathrm{CRI}}^{\mathrm{MT}}=1.5 \times 10^{18} \mathrm{~m}^{-3}$ (for $L=5 \mu \mathrm{m}$ and $d=25 \mathrm{~nm}$ ) satisfy the conditions for the onset of a LQ phase. Highly oriented MTs are on average separated by $0.3 \mu \mathrm{m}$ which means small repulsive forces between MTs prevail. This is consistent with LQ behavior of MTs within oriented "curtains". The present work demonstrates that under earth's gravitational field, MTs assemblies behave as typical polar LQs.

The direction of gravity breaks the isotropic symmetry of the initially homogenous state that triggers the self-organization process of MTs and induces the overall growth of the MT aggregates, eventually leading to the emergence of form and pattern. Such processes may have played important role in the development of life on earth. This gravity-mediated transition leading to symmetry breaking phenomena, is crucial in the establishment of physical self-organization in biology and may constitute a preliminary step in describing biological pattern formation.

Finally, one may discuss some other effects apart from gravity. MTs may probably be sensitive to other weak external stimuli. For example, effects of electric fields on MTs are quite well known from the work of Unger et al. [26]. The effects of magnetic 
fields are well described by W. Bras and his collaborators [27]. The application of such a weak field at the correct moment could act as a switch to form prespecified microtubular arrangements and subsequently instigate or participate in other biological processes, which means that weak effects can be transduced in the cell and amplified.

\section{References}

1. Papaseit, C., Pochon, N., Tabony, J.: Microtubule self-organization is gravity-dependent. Proc. Natl. Acad. Sci. USA 97, 8364-8368 (2000)

2. de Pablo, P.J., Schaap, I.A.T., MacKintosh, F.C., Schmidt, C.F.: Deformation and collapse of microtubules on the nanometer scale. Phys. Rev. Lett. 91, 098101 (2003)

3. Athenstaedt, H.: Pyroelectric and piezoelectric properties of vertebrates. Ann. N.Y. Acad. Sci, 238, 6894 (1974)

4. Margulis, L., To, L., Chase, D.: Microtubules in prokaryotes. Science 200, 1118-1124 (1978)

5. Hameroff, S.R., Watt, R.C.: Information processing in microtubules. J. Theor. Biol. 98, 549-561/(1982)

6. Kis, A., Kasas, S., Babic, B., Kulik, A.J., Benoît, W., Briggs, G.A.D., Schönenberger, C., Catsicas, S., Forró, L.: Nanomechanics of microtubules. Phys. Rev. Lett. 89, 248101 (2002)

7. Spacelab I Reports. Science 225, 205-235 (1984)

8. Tabony, J., Job, D.: Gravitational symmetry breaking in microtubular dissipative structures. Proc. Natl. Acad. Sci. USA 89, 6948-6952 (1992)

9. Portet, S., Tuszyński, J.A., Dixon, J.M., Satarić, M.V.: Models of spatial and orientational selforganization of microtubules under the influence of gravitational fields. Phys. Rev. E 68, 021903 (2003)

10. Melton, D.A.: Pattern formation during animal development. Science 252, 234-241 (1991)

11. Gerhart, J., Keller, R.: Region-specific cell activities in amphibian gastrulation. Annu. Rev. Cell. Biol. 2, 201-229 (1986)

12. Zisckind, N., Elinson, R.P.: Gravity and microtubules in dorsoventral polarization of the Xenopus Egg Develop. Growth Differ. 32, 575-581 (1990)

13. Beetschen, J.C., Gautier, J.: Heat-shock-induced grey crescent formation in axolotl eggs and oocytes: the role of gravity. Development 100, 599-609 (1987)

14. Malacinski, G.M., Neff, A.W.: The amphibian egg as a model system for analyzing gravity effects. Adv. Space Res. 9, 169-176 (1989)

15. Elinson, R.P., Rowning, B.: A transient array of parallel microtubules in frog eggs: potential tracks for a cytoplasmic rotation that specifies the dorso-ventral axis. Dev. Biol. 128, 185-197 (1988)

16. Driss-Ecole, D., Lefranc, A., Perbal, G.; A polarized cell: the root statocyte. Physiol. Plant. 118, 305-312 (2003)

17. Himmelspach, R., Wymer, C.L., Lioyd, C.W., Nick, P.: Gravity-induced reorientation of cortical microtubules observed in vivo. Plant J. 18, 449-453 (1999)

18. Fischer, K., Schopfer, P.: Physical strain-mediated microtubule reorientation in the epidermis of gravitropically or phototropically stimulated maize coleoptiles. Plant J. 15, 119-123 (1998)

19. Parsons, J.D.: Nematic ordering in a system of rods. Phys. Rev. A 19, 1225-1230 (1979)

20. de Gennes, P.: Polymer Liquid Crystals. Academic Press, New York, (1982)

21. Baulin, N.A., Khokhlov, A.R.: Nematic ordering of rigid rods in a gravitational field. Phys. Rev. E 60, 2973-2977 (1999)

22. Sin-Doo, L.: A numerical investigation of nematic ordering based on a simple hard-rod model. J. Chem. Phys. 87, 4972-4974 (1987)

23. Satarić, M.V., Tuszyński, J.A.: Relationship between the nonlinear ferroelectric and liquid crystal models for microtubules. Phys. Rev. E 67, 011901 (2003)

24. Tuszyński, J.A., Satarić, M.V., Portet, S., Dixon, J.M.: Gravitational symmetry breaking leads to a polar liquid crystal phase of microtubules in vitro. J. Biol. Phys. 31, 477-486 (2005)

25. Baulin, V.A.: Self-assembled aggregates in the gravitational field: growth and nematic order. J. Chem. Phys. 119, 2874-2885 (2003)

26. Stracke, R., Böhm, K.J., Wollweber, L., Tuszyński, J.A., Unger, E.: Analysis of the migration behaviour of single microtubules in electric fields. Biochem. Biophys. Res. Commun. 293, 602-609 (2002)

27. Bras, W., Diakun, G.P., Diaz, J.F., Maret, G., Kramer, H., Bordas, J., Medrano, F.J.: The Susceptibility of pure tubulin to high magnetic fields: a magnetic birefringence and X-ray fiber diffraction study. Biophys. J. 74, 1509-1521 (1998) 